\title{
Controlling Chaos through Period-Doubling Bifurcations in Attitude Dynamics for Power Systems
}

\author{
Shun-Chang Chang (i) \\ Department of Mechanical and Automation Engineering, Da-Yeh University, Changhua 51591, Taiwan \\ Correspondence should be addressed to Shun-Chang Chang; changsc@mail.dyu.edu.tw
}

Received 28 September 2020; Revised 10 November 2020; Accepted 18 November 2020; Published 4 December 2020

Academic Editor: José A. F. O. Correia

Copyright (C) 2020 Shun-Chang Chang. This is an open access article distributed under the Creative Commons Attribution License, which permits unrestricted use, distribution, and reproduction in any medium, provided the original work is properly cited.

\begin{abstract}
This paper addresses the complex nonlinear dynamics involved in controlling chaos in power systems using bifurcation diagrams, time responses, phase portraits, Poincaré maps, and frequency spectra. Our results revealed that nonlinearities in power systems produce period-doubling bifurcations, which can lead to chaotic motion. Analysis based on the Lyapunov exponent and Lyapunov dimension was used to identify the onset of chaotic behavior. We also developed a continuous feedback control method based on synchronization characteristics for suppressing of chaotic oscillations. The results of our simulation support the feasibility of using the proposed method. The robustness of parametric perturbations on a power system with synchronization control was analyzed using bifurcation diagrams and Lyapunov stability theory.
\end{abstract}

\section{Introduction}

The characteristics of power systems are inherently nonlinear; this is due to the nonlinearity of synchronous generators. Power systems are usually described using a nonlinear dynamical system of equations and system parameters, where any change in one of the parameters can destabilize the entire system, resulting in chaotic motion and eventual voltage collapse as well as catastrophic blackout. Various researchers have studied voltage collapse in electric power systems [1-5].

Chaotic behavior must be accepted in some situations; however, it is normally undesirable, as it degrades performance and restricts the operating range of electric and mechanical devices. Beginning with Ott et al. [6], researchers $[7,8]$ have sought to develop control methods for converting chaotic motions into periodic orbits or steady states. Researchers $[9,10]$ have made particular progress in single-machine infinite bus systems (SMIB) by applying the classical generator model. In this work, we sought to convert chaotic behavior into periodic motion in dynamic power systems based on multiple machines. The synchronization of chaos has received considerable attention [11-16]. Usually, a periodic system is referred to as the master (drive) system and a chaotic system as the slave (response) system. The principle underlying synchronization is the control of a slave system using the output of a master system, such that the output of the slave system follows the output of the master system asymptotically. Continuous feedback control methods based on the properties of synchronization, such as the scheme developed by Pyragas [17] and Kapitaniak [18], can be used to convert chaotic motion into stable motion by using feedback in conjunction with a periodic external force. This approach has been used in numerous chaotic systems $[19,20]$. Chang and $\mathrm{Hu}[19]$ used synchronization to lessen chaotic behavior in an automotive suspension system. Chang and Lue [20] used synchronization to achieve stable equilibrium in a magnetic levitation system.

Modern nonlinear theories of bifurcation and chaos have been developed to study nonlinear systems, and numerous studies on nonlinear dynamics in power systems have been published [21-27]. In this work, we employed bifurcation diagrams, phase portraits, Poincaré maps, and frequency spectra to examine the rich nonlinear dynamics of power systems. We also adopted advanced algorithms to compute Lyapunov exponents of smooth dynamical 
systems [28] to identify instances of chaos. Then, we developed a continuous feedback control method based on synchronization characteristics to suppress chaotic oscillations in power systems. We then performed simulations to assess the feasibility and efficiency of the scheme. Finally, the design of the feedback controller was validated through the application of optimal control and Lyapunov stability theories, guaranteeing the global stability of nonlinear error systems [29-31].

This paper is organized as follows. Section 2 describes a power system model involving three synchronous generators with a resistive load configuration. Section 3 describes the complex dynamic behavior of a power system, which was elucidated using numerical analysis methods, such as bifurcation diagrams, phase portraits, Poincaré maps, and frequency spectra. Section 4 presents the Lyapunov exponent used to determine whether the system exhibits chaotic motion. A synchronization control technique for controlling chaos in power systems is described in Section 5. Section 6 reveals the effects of parameter errors on the performance of the proposed synchronization control system. Conclusions are outlined in Section 7.

\section{Mathematical Modeling of Swing Equations for Three Machines}

Synchronous generators are the most critical energy sources in power systems; however, they are also the source of most dynamic fluctuations. A power system model involving three synchronous generators with a resistive load configuration is considered, as illustrated in Figure 1. Following [32, 33], the dynamics of this nonlinear power system can be expressed as follows:

$$
\begin{aligned}
\dot{\delta}_{1} & =\omega_{1}, \\
M_{1} \dot{\omega}_{1} & =-D_{1} \omega_{1}+P_{1}-F_{12} \sin \left(\delta_{1}-\delta_{2}\right)-F_{13} \sin \left(\delta_{1}-\delta_{3}\right), \\
\dot{\delta}_{2} & =\omega_{2}, \\
M_{2} \dot{\omega}_{2} & =-D_{2} \omega_{2}+P_{2}-F_{21} \sin \left(\delta_{2}-\delta_{1}\right)-F_{23} \sin \left(\delta_{2}-\delta_{3}\right), \\
\dot{\delta}_{3} & =\omega_{3}, \\
M_{3} \dot{\omega}_{3} & =-D_{3} \omega_{3}+P_{3}-F_{31} \sin \left(\delta_{3}-\delta_{1}\right)-F_{32} \sin \left(\delta_{3}-\delta_{2}\right) .
\end{aligned}
$$

Now, a special case is considered in which swing equations are applied for dynamics analysis of the three machines. We assume that Machine 1 has large inertia, where $M_{1}=\left(\bar{M}_{1} / \varepsilon\right), \varepsilon \ll 1$. The transmission line joining Machines 2 and 3 is shorter than the other lines. Similarly, the external power $P_{1}$ is proportionally larger: $P_{1}=\bar{P}_{1} / \varepsilon$. Under these assumptions, the conservative swing equations for three machines can be written as follows:

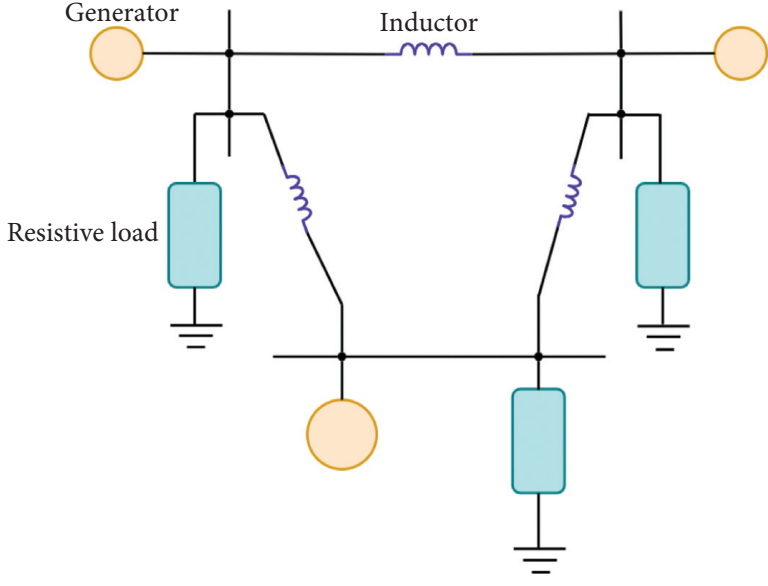

FIgURE 1: Schematic diagram of the power system.

$$
\begin{aligned}
\dot{\delta}_{1} & =\omega_{1}, \\
\bar{M}_{1} \dot{\omega}_{1} & =-\varepsilon D_{1} \omega_{1}+\bar{P}_{1}-\varepsilon F_{12} \sin \left(\delta_{1}-\delta_{2}\right)-\varepsilon F_{13} \sin \left(\delta_{1}-\delta_{3}\right), \\
\dot{\delta}_{2} & =\omega_{2}, \\
M_{2} \dot{\omega}_{2} & =-D_{2} \omega_{2}+P_{2}-F_{21} \sin \left(\delta_{2}-\delta_{1}\right)-F_{23} \sin \left(\delta_{2}-\delta_{3}\right), \\
\dot{\delta}_{3} & =\omega_{3}, \\
M_{3} \dot{\omega}_{3} & =-D_{3} \omega_{3}+P_{3}-F_{31} \sin \left(\delta_{3}-\delta_{1}\right)-F_{32} \sin \left(\delta_{3}-\delta_{2}\right) .
\end{aligned}
$$

In accordance with $[32,33], \delta_{1}$ can be expressed as follows:

$$
\delta_{1}=-\varepsilon \mu_{2} \delta_{2}-\varepsilon \mu_{3} \delta_{3},
$$

where $\mu_{2}=\left(M_{2} / \bar{M}_{1}\right)$ and $\mu_{3}=\left(M_{3} / \bar{M}_{1}\right)$.

Substituting equation (3) into equations (2a)-(2f), we can construct an autonomous differential equation for $\delta_{2}, \delta_{3}$, $\omega_{2}$, and $\omega_{3}$, thereby eliminating $\delta_{1}$ and $\omega_{1}$ as follows:

$$
\begin{aligned}
\dot{\delta}_{2}= & \omega_{2}, \\
\dot{\omega}_{2}= & -D_{2} \omega_{2}+\bar{\alpha}_{2}-\beta_{21} \sin \left[\delta_{2}\left(1+\varepsilon \mu_{2}\right)+\varepsilon \mu_{3} \delta_{3}\right] \\
& -\beta_{23} \sin \left(\delta_{2}-\delta_{3}\right), \\
\dot{\delta}_{3}= & \omega_{3}, \\
\dot{\omega}_{3}= & -D_{3} \omega_{3}+\bar{\alpha}_{3}-\beta_{31} \sin \left[\delta_{2}\left(1+\varepsilon \mu_{2}\right)+\varepsilon \mu_{3} \delta_{3}\right] \\
& -\beta_{32} \sin \left(\delta_{3}-\delta_{2}\right),
\end{aligned}
$$

where $\bar{\alpha}_{2}=\left(P_{2} / M_{2}\right), \quad \beta_{21}=\left(F_{21} / M_{2}\right), \quad \beta_{23}=\left(F_{23} / M_{2}\right)$, $\bar{\alpha}_{3}=\left(P_{3} / M_{3}\right), \beta_{31}=\left(F_{31} / M_{3}\right)$, and $\beta_{32}=\left(F_{32} / M_{3}\right)$.

For the sake of convenience, we let $\varepsilon=0$ and simplify equations (4a)-(4d) as follows: 


$$
\begin{aligned}
& \dot{\delta}_{2}=\omega, \\
& \dot{\omega}_{2}=-D_{2} \omega_{2}+\bar{\alpha}_{2}-\beta_{21} \sin \left(\delta_{2}\right)-\beta_{23} \sin \left(\delta_{2}-\delta_{3}\right), \\
& \dot{\delta}_{3}=\omega_{3}, \\
& \dot{\omega}_{3}=-D_{3} \omega_{3}+\bar{\alpha}_{3}-\beta_{31} \sin \left(\delta_{3}\right)-\beta_{32} \sin \left(\delta_{3}-\delta_{2}\right) .
\end{aligned}
$$

In accordance with $[32,33], \bar{\alpha}_{k}$ can be expressed as follows:

$$
\bar{\alpha}_{k}=\bar{P}_{k}-K_{f} \omega_{k}, \quad k=2,3,
$$

where $P_{k}$ indicates the constant real power, $K_{f}=L_{k} / M_{k}$, and $L_{k}$ is a load-frequency coefficient. Substituting equation (6) into equations $(5 \mathrm{a})-(5 \mathrm{~d})$, we obtain

$$
\begin{aligned}
& \dot{\delta}_{2}=\omega_{2}, \\
& \dot{\omega}_{2}=-D_{2} \omega_{2}+\bar{P}_{2}-K_{f} \omega_{2}-\beta_{21} \sin \left(\delta_{2}\right)-\beta_{23} \sin \left(\delta_{2}-\delta_{3}\right), \\
& \dot{\delta}_{3}=\omega_{3}, \\
& \dot{\omega}_{3}=-D_{3} \omega_{3}+\bar{P}_{3}-K_{f} \omega_{3}-\beta_{31} \sin \left(\delta_{3}\right)-\beta_{32} \sin \left(\delta_{3}-\delta_{2}\right) .
\end{aligned}
$$

Assuming $y_{1}=\delta_{2}, y_{2}=\omega_{2}, y_{3}=\delta_{3}$, and $y_{4}=\omega_{3}$ are the state variables, such that the state-space model of the swing equation can be written as follows:

$$
\begin{aligned}
& \dot{y}_{1}=y_{2}, \\
& \dot{y}_{2}=\bar{P}_{2}-\left(D_{2}+K_{f}\right) y_{2}-\beta_{21} \sin \left(y_{1}\right)-\beta_{23} \sin \left(y_{1}-y_{3}\right), \\
& \dot{y}_{3}=y_{4}, \\
& \dot{y}_{4}=\bar{P}_{3}-\left(D_{3}+K_{f}\right) y_{4}-\beta_{31} \sin \left(y_{3}\right)-\beta_{32} \sin \left(y_{3}-y_{1}\right) .
\end{aligned}
$$

The numerical values of all parameters in equations (8a)-(8d) are listed in Table 1 [33].

\section{Power System Characteristics}

Numerical simulations based on equations (8a)-(8d) were used to elucidate the characteristics of the power system. The commercial package DIVPRK of IMSL was used to write a series of FORTRAN subroutines to solve the ordinary differential equation (ODE) [34]. The resulting bifurcation diagram displayed in Figure 2 shows that the first perioddoubling bifurcation occurred at approximately $K_{f}=0.078$, with chaotic motion appearing at approximately $K_{f}=0.0109$. More details on the various responses exhibited by the system are shown in Figures 3-6. Each response type is characterized using a phase portrait, Poincaré map, and frequency spectrum. When the parameter $K_{f}>0.078$, the
TABle 1: Physical parameters of a power system.

\begin{tabular}{lc}
\hline Parameter & Value \\
\hline$\beta_{21}$ & -2 \\
$\beta_{23}$ & -1 \\
$\beta_{31}$ & -1 \\
$\beta_{32}$ & -1 \\
$D_{2}$ & 0.2 \\
$D_{3}$ & 0.5 \\
$\bar{P}_{2}$ & 0.3 \\
$\bar{P}_{3}$ & 0.5 \\
\hline
\end{tabular}

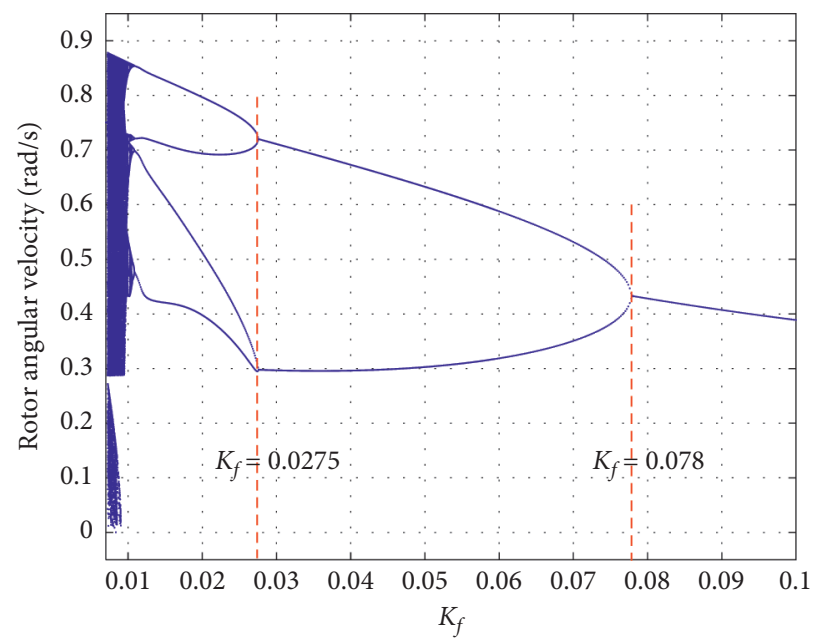

Figure 2: Bifurcation diagram of the power system.

equilibrium point of equations (8a)-(8d) is stable, revealing the absence of chatter vibration. Figures 3(a)-3(c) present period-one motion. Figures 4(a)-4(c) illustrate that a cascade of period-doubling bifurcations caused a series of subharmonic components, which resulted in bifurcations with new frequency components at $\Omega / 2,3 \Omega / 2,5 \Omega / 2, \ldots$, $(2 n-1) \Omega / 2$. Decreasing $K_{f}$ to $K_{f}=0.0275$ caused the first period-4 bifurcation, as displayed in Figures 5(a)-5(c). Further decreasing $K_{f}$ resulted in a cascade of period-doubling bifurcations, leading to chaos. The resulting chatter vibration was sufficient to cause a voltage collapse. We used Poincaré maps and frequency spectra to characterize the chaotic behavior. Poincaré maps present chaotic motion as an infinite set of points (a strange attractor). Strange attractors and continuous Fourier spectra are strong indicators of chaos. Figures 6(a)-6(c) illustrate the chaotic behavior in detail.

\section{Analysis of Chaotic Phenomena in Power Systems}

In this section, Lyapunov exponents are used to overcome the limitations of the schemes used in Section 3 to identify the occurrence of chaotic motion. Every dynamic system involves a spectrum of Lyapunov exponents $(\lambda)$ [28], which indicate changes in the length, area, and volume of a phase space. Determining whether a system exhibits characteristics 

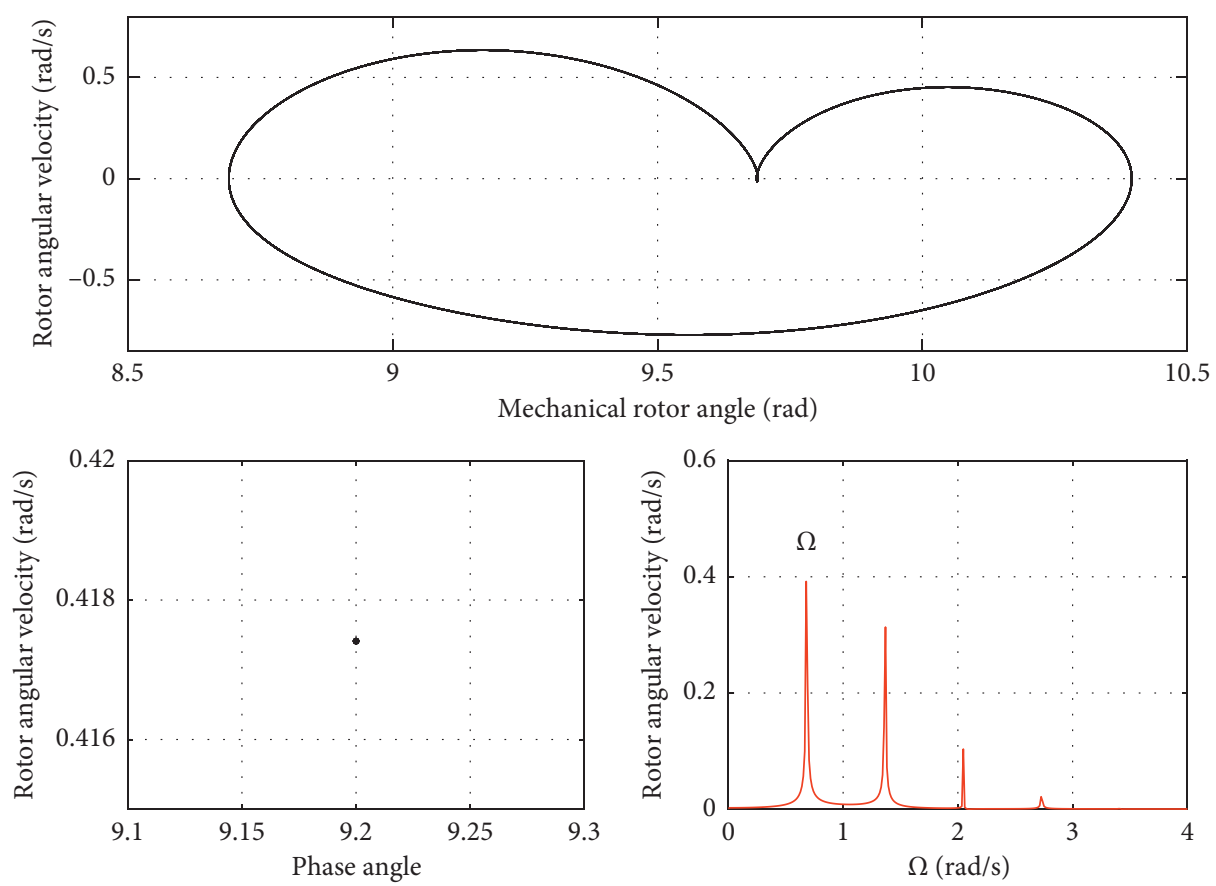

FIgURe 3: Period-1 motion at $K_{f}=0.085$ : (a) phase portrait; (b) Poincaré map; (c) power spectrum.
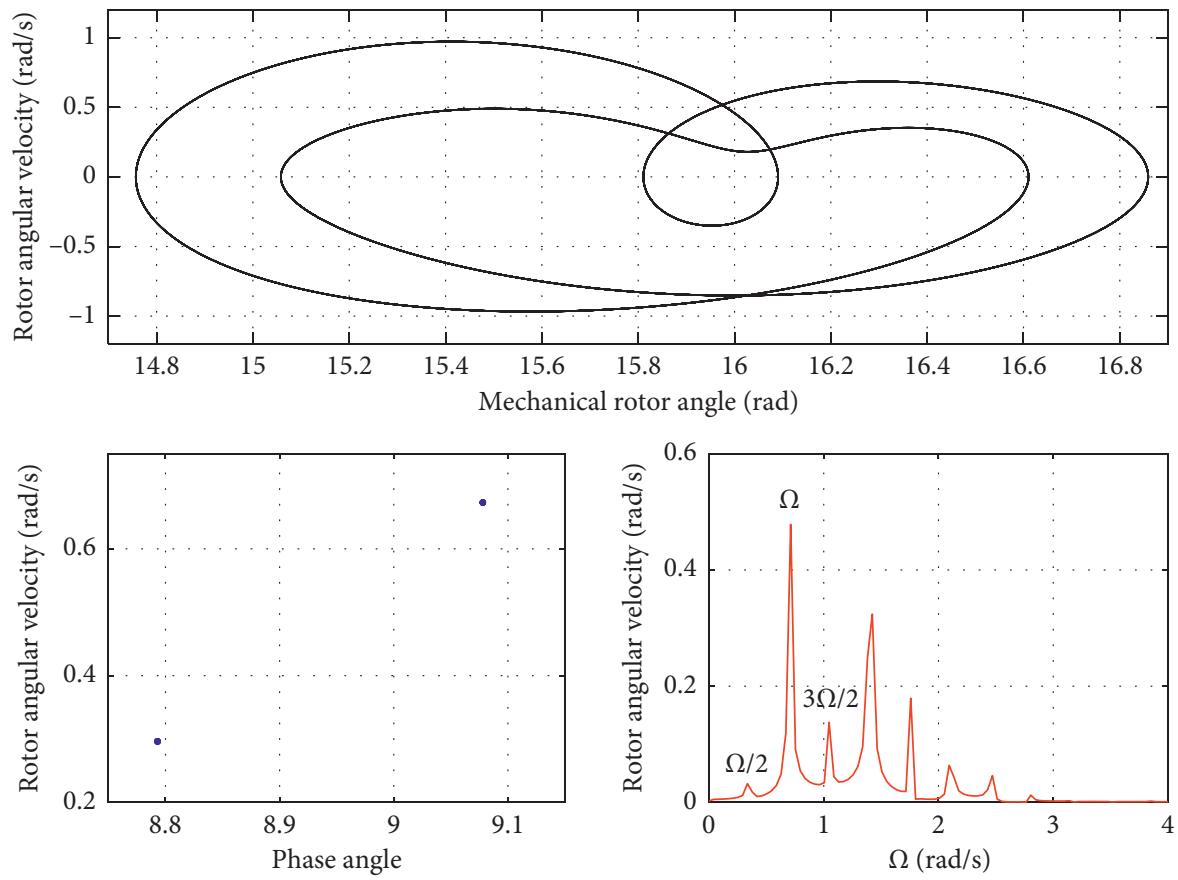

Figure 4: Period-2 motion at $K_{f}=0.04$ : (a) phase portrait; (b) Poincaré map; (c) power spectrum.

of chaos only requires calculation of the largest Lyapunov exponent to determine whether, on average, the nearby trajectories diverge $(\lambda>0)$ or converge $(\lambda<0)$. Chaotic motion exhibits at least one positive Lyapunov exponent within a bounded system, whereas periodic motion exhibits no positive Lyapunov exponents.

In the current study, we adopted the algorithm proposed by Wolf et al. [28] for the calculation of Lyapunov exponents. Figure 7 illustrates the evolution of the largest Lyapunov exponent, in which the onset of chaotic motion occurs at approximately $K_{f}=0.0109$. At point $P_{3}$, the gradual reduction in $K_{f}$ causes the sign of the largest Lyapunov exponent to change from negative to positive. At points $P_{1}$ and $P_{2}$, the largest Lyapunov exponent approached zero, beyond which the system is susceptible to bifurcation. However, the Lyapunov exponent at these points provides no information pertaining to the class of bifurcation. We must therefore consult the bifurcation diagram presented in Figure 2. A 

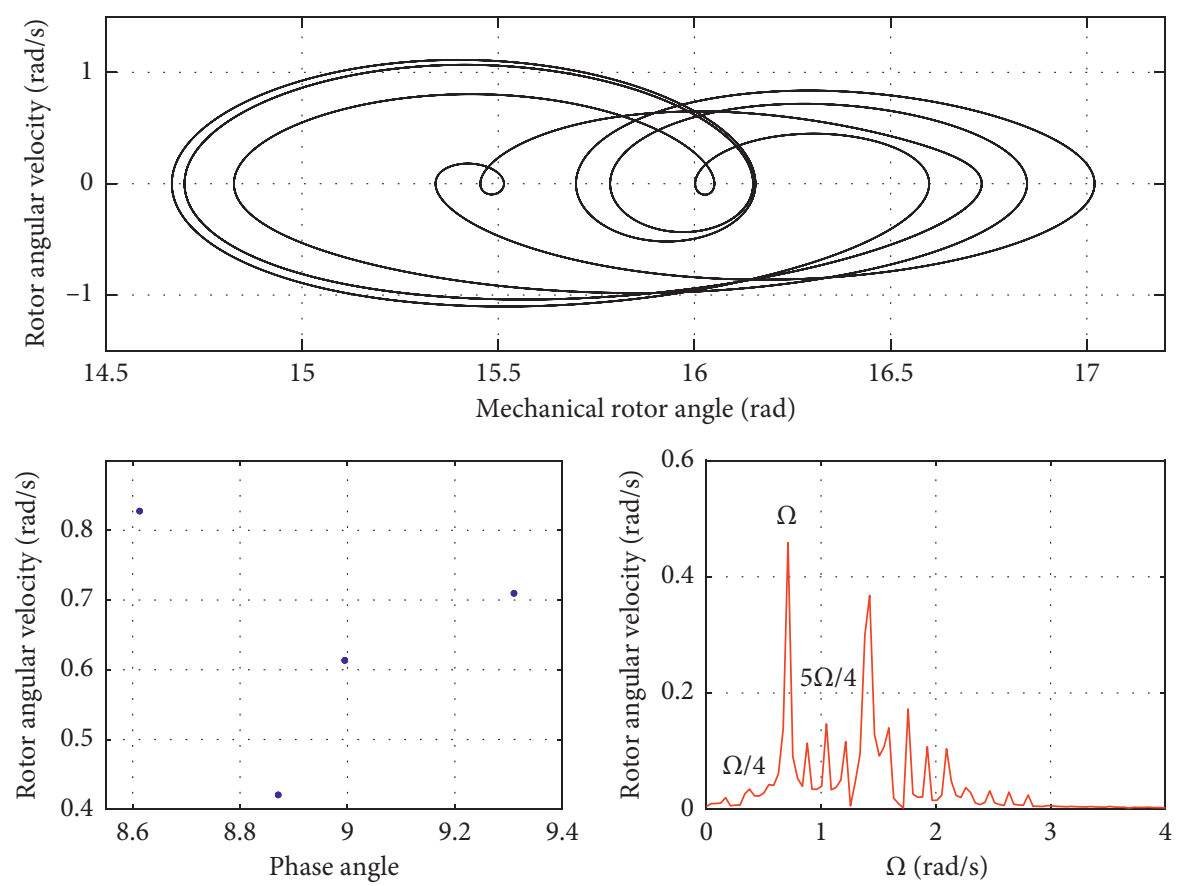

Figure 5: Period-4 motion at $K_{f}=0.015$ : (a) phase portrait; (b) Poincaré map; (c) power spectrum.
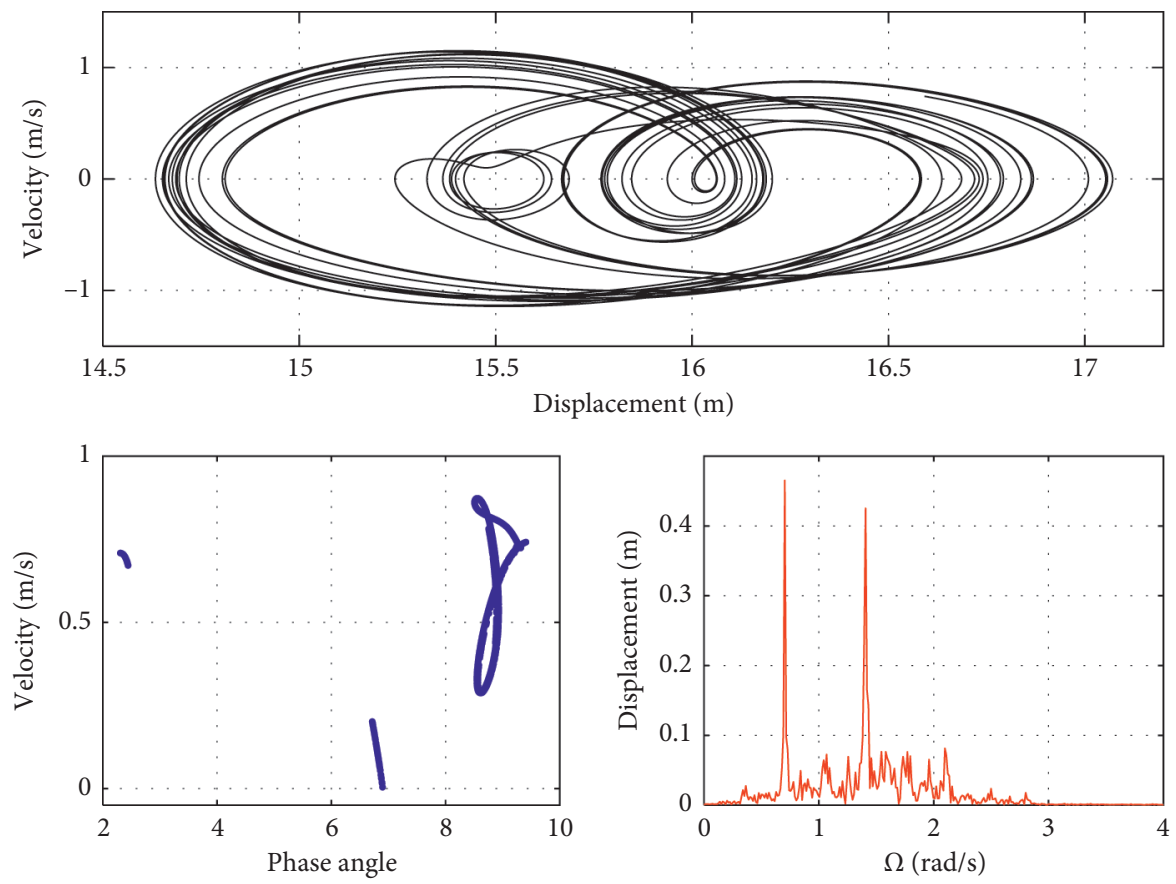

FIgure 6: Chaotic orbit at $K_{f}=0.008$ : (a) phase portrait; (b) Poincaré map; (c) power spectrum.

comparison of Figures 7 and 2 revealed that period- 2 and period-4 bifurcations occur at $P_{1}$ and $P_{2}$, respectively. When $K_{f}=0.09$, the Lyapunov exponents obtained using equations (8a)-(8d) are $\quad \lambda_{1}=-0.000084, \quad \lambda_{2}=-0.0157341$, $\lambda_{3}=-0.5120906$, and $\lambda_{4}=-0.5977934$. The negative value $\lambda_{1}+\lambda_{2}+\lambda_{3}+\lambda_{4}=-1.1257021$ indicates that the power system exhibits stable periodic motion in its current state. Denoting $\lambda_{1} \geq \cdots \geq \lambda_{n}$ as the Lyapunov exponents of a dynamical system, Kaplan and Yorke [35] provided a means by which to estimate the Lyapunov dimension $d_{\mathrm{L}}$ : 


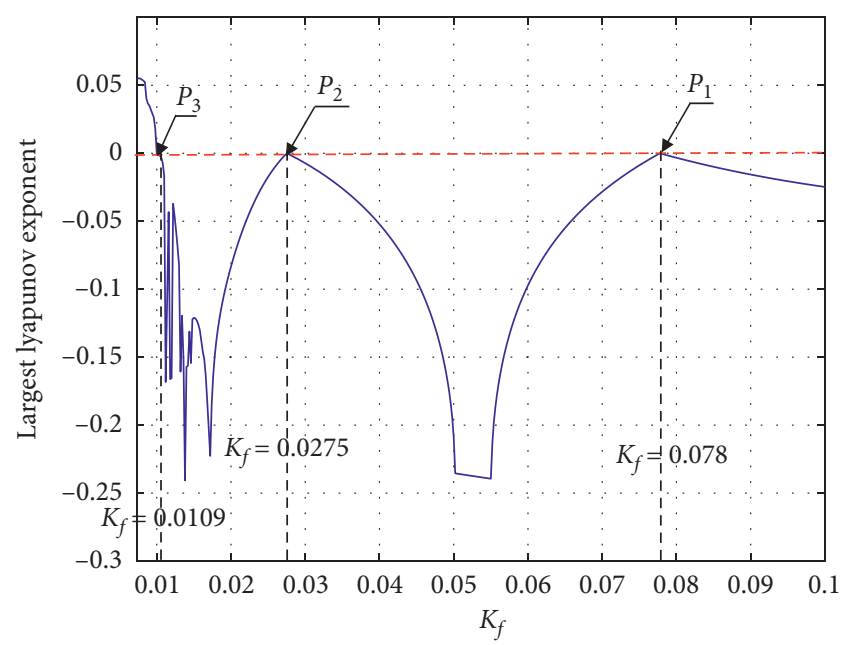

Figure 7: Maximum Lyapunov exponent vs. $K_{f}$.

$$
d_{\mathrm{L}}=j+\frac{1}{\left|\lambda_{j+1}\right|} \sum_{i=1}^{j} \lambda_{i}
$$

where

$$
\begin{aligned}
& \sum_{i=1}^{j} \lambda_{i}>0, \\
& \sum_{i=1}^{j+1} \lambda_{i}<0 .
\end{aligned}
$$

Periodic orbit is indicated by an integer-valued Lyapunov dimension, whereas the noninteger value of the Lyapunov dimension indicates chaotic motion. When $K_{f}=0.09$, using the technique in equations (8a)-(8d), returned a Lyapunov dimension of $d_{\mathrm{L}}=1$, which indicates a system undergoing periodic motion. When $K_{f}$ decreased beyond the bifurcation point $P_{3}$ to $K_{f}=0.009$, the Lyapunov exponents were $\lambda_{1}=0.0340716, \quad \lambda_{2}=-0.0000068$, $\lambda_{3}=-0.3985904$, and $\lambda_{4}=-0.5270599$, and the Lyapunov dimension was $d_{\mathrm{L}}=2.0854$. In this situation, the largest Lyapunov exponent was positive and the Lyapunov dimension was a noninteger, indicating that the power system was undergoing chaotic motion.

\section{Controlling Chaos through Synchronization}

Although there are some benefits to identifying chaotic systems, the ultimate objective is to assume control over such systems. Avoiding chaos in dynamic systems requires transformation of chaotic motion into periodic motion. Pyragas [17] and Kapitaniak [18] proposed a simple yet effective time-continuous control method for the conversion of chaotic motion into periodic motion using time-continuous perturbation with a feedback mechanism. Figure 8 plots the control system proposed in this study, which comprises a feedback-controlled loop with external periodic perturbation. This method is explained briefly in the following paragraph.

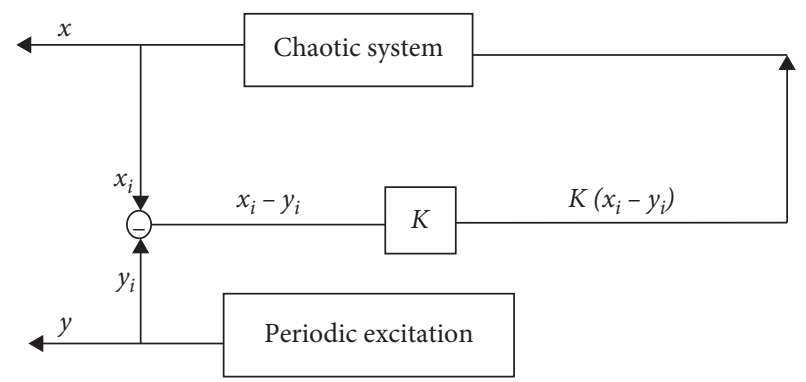

FIgURE 8: Block diagram illustrating the proposed continuous chaos control scheme.

Consider the following $n$-dimensional dynamic system:

$$
\begin{aligned}
& \dot{x}=P(x)+F(t), \\
& \dot{y}=Q(y),
\end{aligned}
$$

where $x(t), y(t) \in R^{n}$ is the state vector and $F(t)$ is the input signal. Equation (11) without an input signal $(F(t)=0)$ is assumed to have a strange attractor, whereas periodic motion is observed in equation (12). Through the strategy presented in Figure 8, the periodic system (i.e., drive system) is synchronized with the chaotic system (i.e., response system). The difference between signals $y(t)$ and $x(t)$ yields the following control signal:

$$
F(t)=K[x(t)-y(t)],
$$

where $K$ indicates the feedback gain.

When $K_{f}=0.09$ is selected as the drive system, equations (14a)-(14d) reveal period-1 motion.

$$
\begin{aligned}
& \dot{y}_{1}=y_{2} \\
& \dot{y}_{2}=\bar{P}_{2}-\left(D_{2}+K_{f}\right) y_{2}-\beta_{21} \sin \left(y_{1}\right)-\beta_{23} \sin \left(y_{1}-y_{3}\right) \\
& \dot{y}_{3}=y_{4} \\
& \dot{y}_{4}=\bar{P}_{3}-\left(D_{3}+K_{f}\right) y_{4}-\beta_{31} \sin \left(y_{3}\right)-\beta_{32} \sin \left(y_{3}-y_{1}\right)
\end{aligned}
$$

When $K_{f}=0.008$ is selected in the response system, equations (15a)-(15d) reveal chaotic motion.

$$
\begin{aligned}
& \dot{x}_{1}=x_{2}, \\
& \dot{x}_{2}=\bar{P}_{2}-\left(D_{2}+K_{f}\right) x_{2}-\beta_{21} \sin \left(x_{1}\right)-\beta_{23} \sin \left(x_{1}-x_{3}\right) \\
& \dot{x}_{3}=x_{4}, \\
& \dot{x}_{4}=\bar{P}_{3}-\left(D_{3}+K_{f}\right) x_{4}-\beta_{31} \sin \left(x_{3}\right)-\beta_{32} \sin \left(x_{3}-x_{1}\right)
\end{aligned}
$$


Synchronizing equations (14a)-(14d) and (15a)-(15d) involves introducing the control signal in equation (13) into equations (15a)-(15d) as feedback control, which yields the following coupled system:

$$
\begin{aligned}
\dot{x}_{1}= & x_{2}, \\
\dot{x}_{2}= & \bar{P}_{2}-\left(D_{2}+K_{f}\right) x_{2}-\beta_{21} \sin \left(x_{1}\right)-\beta_{23} \sin \left(x_{1}-x_{3}\right) \\
& +K\left(x_{2}-y_{2}\right), \\
\dot{x}_{3}= & x_{4}, \\
\dot{x}_{4}= & \bar{P}_{3}-\left(D_{3}+K_{f}\right) x_{4}-\beta_{31} \sin \left(x_{3}\right)-\beta_{32} \sin \left(x_{3}-x_{1}\right) \\
& +K\left(x_{3}-y_{3}\right) .
\end{aligned}
$$

Equations (16a)-(16d) describe chaotic motion when $K=0$ and $K_{f}=0.008$. The feedback gain $K$ is adjusted between 0.0 and -1.0 to convert the dynamics of the system from chaotic motion into periodic motion. Figure 9 presents the resulting bifurcation diagram, which comprehensively explains the dynamic behavior of the system over a range of feedback gains. Stable periodic motion appears when $K$ decreases below 0.005 . Period-doubling bifurcations appear when $K$ decreases to between approximately -0.135 and -0.375 . A further decrease in $K$ beyond -0.375 results in period- 1 motion. The efficacy of the proposed system in controlling chaos was demonstrated by the application of a synchronization control signal $(K=-0.8)$ after a period of $100 \mathrm{~s}$, as displayed in Figure 10.

\section{Effects of Parametric Perturbations on Power Systems Using Synchronization Control}

We sought to understand the effects of parameter errors on the performance of the proposed synchronization control system by adding a sinusoidal perturbation directly to parameters $\beta_{21}, \beta_{23}, \beta_{31}, \beta_{32}, D_{2}, D_{3}, \bar{P}_{2}$, and $\bar{P}_{3}$ in the periodic excitation (drive system). Assume that equations (14a)-(14d) are representative of the drive system; then, the corresponding controlled response system is given by

$$
\begin{aligned}
\dot{z}_{1}= & z_{2}+u_{1}, \\
\dot{z}_{2}= & \bar{P}_{2}(1+\varepsilon \sin (\omega t))-\left(D_{2}(1+\varepsilon \sin (\omega t))+K_{f}\right) z_{2} \\
& -\beta_{21}(1+\varepsilon \sin (\omega t)) \sin \left(z_{1}\right) \\
& -\beta_{23}(1+\varepsilon \sin (\omega t)) \sin \left(z_{1}-z_{3}\right)+u_{2},
\end{aligned}
$$

$$
\dot{z}_{3}=z_{4}+u_{3}
$$

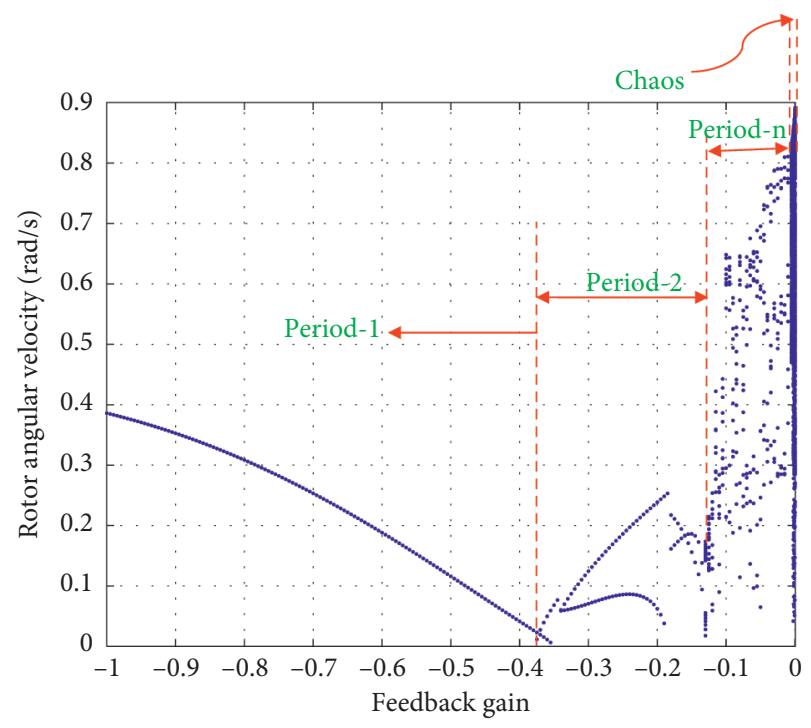

FIgURE 9: Bifurcation diagram of the system with synchronization control, where $K$ denotes feedback gain.

$$
\begin{aligned}
\dot{z}_{4}= & \bar{P}_{3}(1+\varepsilon \sin (\omega t))-\left(D_{3}(1+\varepsilon \sin (\omega t))+K_{f}\right) z_{4} \\
& -\beta_{31}(1+\varepsilon \sin (\omega t)) \sin \left(z_{3}\right) \\
& -\beta_{32}(1+\varepsilon \sin (\omega t)) \sin \left(z_{3}-z_{1}\right)+u_{4},
\end{aligned}
$$

where $\varepsilon$ is the amplitude of the perturbation and $\omega$ is the angular frequency.

Subtracting equations (14a)-(14d) from equations (17a)-(17d), we obtain the following error equation:

$$
\begin{aligned}
\dot{e}_{1}= & e_{2}+u_{1}, \\
\dot{e}_{2}= & \bar{P}_{2} \varepsilon \sin (\omega t)-\left(D_{2} \varepsilon \sin (\omega t)+K_{f}\right) e_{2} \\
& -\beta_{21} \varepsilon \sin (\omega t) \sin \left(e_{1}\right) \\
& -\beta_{23} \varepsilon \sin (\omega t) \sin \left(e_{1}-e_{3}\right)+u_{2}, \\
\dot{e}_{3}= & e_{4}+u_{3}, \\
\dot{e}_{4}= & \bar{P}_{3} \varepsilon \sin (\omega t)-\left(D_{3} \varepsilon \sin (\omega t)+K_{f}\right) e_{4} \\
& -\beta_{31} \varepsilon \sin (\omega t) \sin \left(e_{3}\right) \\
& -\beta_{32} \varepsilon \sin (\omega t) \sin \left(e_{3}-e_{1}\right)+u_{4},
\end{aligned}
$$

where $\quad e_{1}=z_{1}-y_{1}, \quad e_{2}=z_{2}-y_{2}, \quad e_{3}=z_{3}-y_{3}, \quad$ and $e_{4}=z_{4}-y_{4}$.

Considering a Lyapunov function for equations (18a)-(18d),

$$
V(e)=\frac{1}{2} e^{T} e .
$$

We obtain the first derivative of $V(e)$ as follows:

$$
\dot{V}(e)=e_{1} \dot{e}_{1}+e_{2} \dot{e}_{2}+e_{3} \dot{e}_{3}+e_{4} \dot{e}_{4} .
$$



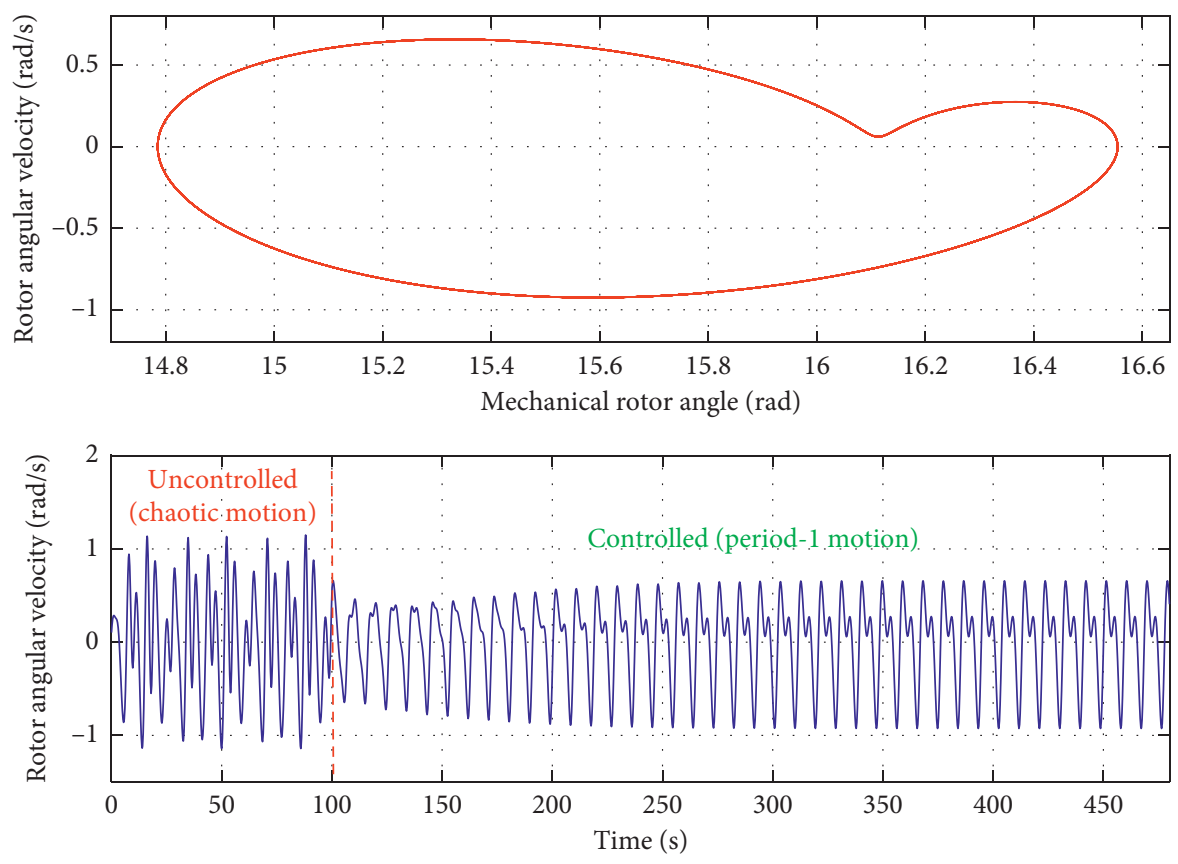

Figure 10: Transforming chaotic motion into a period-1 orbit, where $K=-0.8, K_{f}=0.008$, and a synchronization control signal is introduced after $100 \mathrm{~s}$ : (a) controlled orbit and (b) time history.
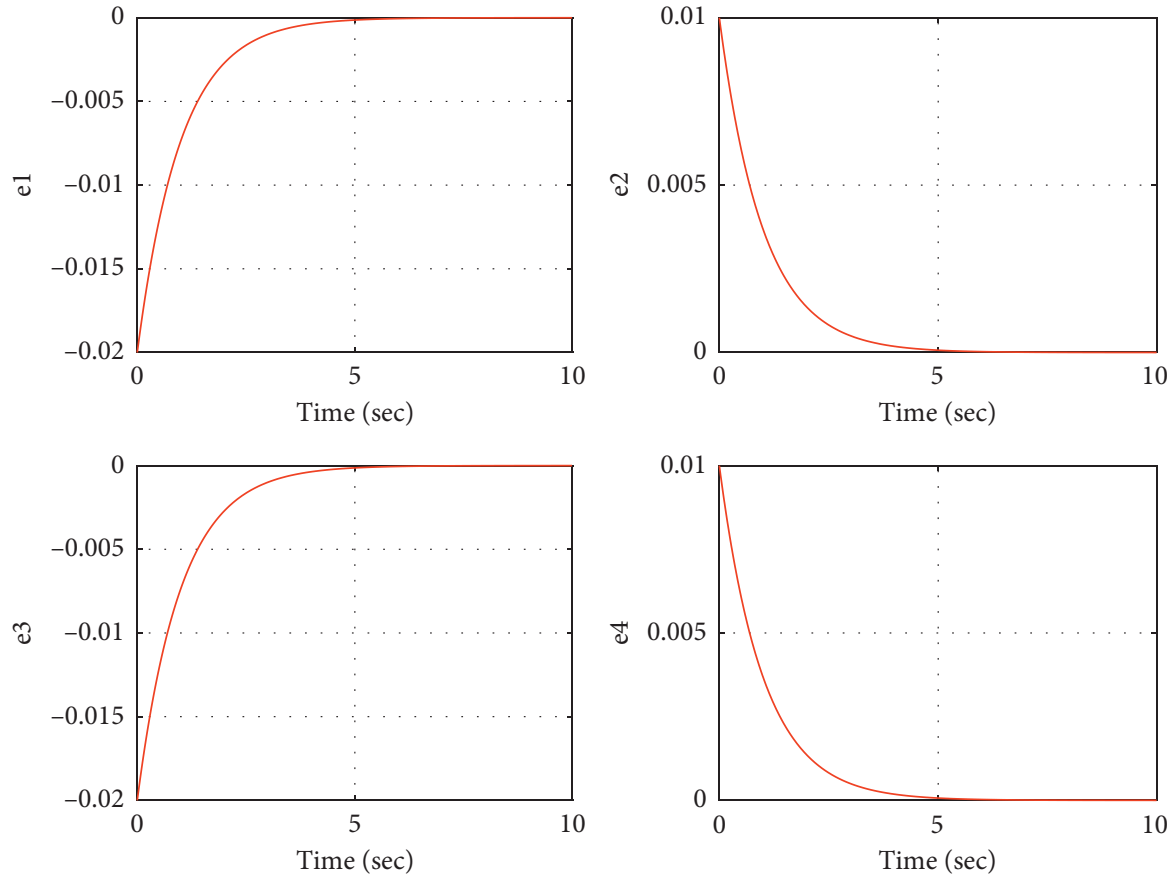

Figure 11: Dynamics of synchronization errors for the drive and response systems: (a) $e_{1}=z_{1}-x_{1}$, (b) $e_{2}=z_{2}-x_{2}$, (c) $e_{3}=z_{3}-x_{3}$, and (d) $e_{4}=z_{4}-x_{4}$. 


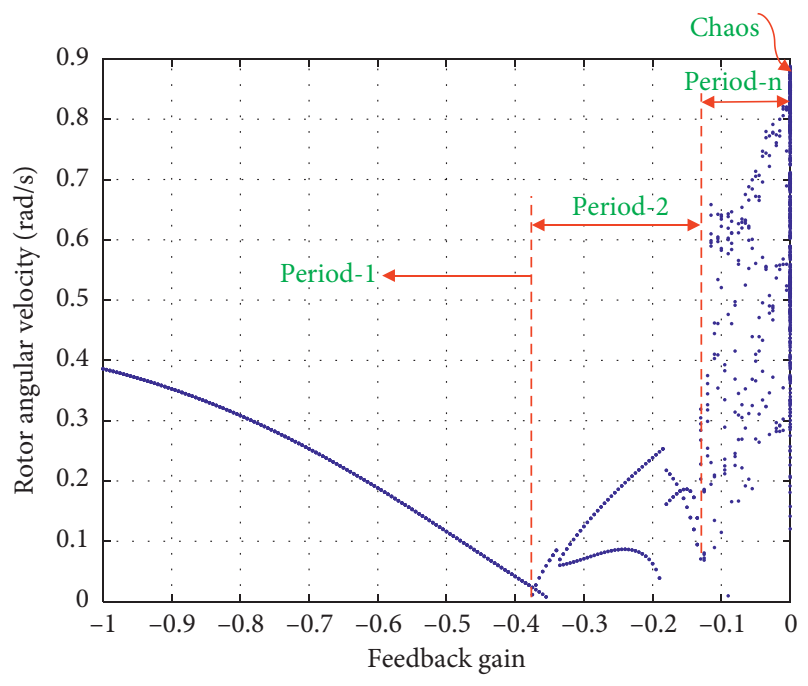

Figure 12: Bifurcation diagram of a system controlled through synchronization under the following perturbed parameters: $\beta_{21}+\beta_{21} \varepsilon \sin \omega t, \beta_{23}+\beta_{23} \varepsilon \sin \omega t, \beta_{31}+\beta_{31} \varepsilon \sin \omega t, \beta_{32}+\beta_{32} \varepsilon \sin \omega t$, $D_{3}+D_{3} \varepsilon \sin \omega t, \bar{P}_{2}+\bar{P}_{2} \varepsilon \sin \omega t$, and $\bar{P}_{3}+\bar{P}_{3} \varepsilon \sin \omega t$.

Therefore, if we select

$$
\begin{aligned}
u_{1}= & -e_{1}-e_{2} \\
u_{2}= & -e_{2}-\bar{P}_{2} \varepsilon \sin (\omega t)+\left(D_{2} \varepsilon \sin (\omega t)+K_{f}\right) e_{2} \\
& +\beta_{21} \varepsilon \sin (\omega t) \sin \left(e_{1}\right)+\beta_{23} \varepsilon \sin (\omega t) \sin \left(e_{1}-e_{3}\right)
\end{aligned}
$$

$$
\begin{aligned}
u_{3}= & -e_{3}-e_{4} \\
u_{4}= & -e_{4}-\bar{P}_{3} \varepsilon \sin (\omega t)+\left(D_{3} \varepsilon \sin (\omega t)+K_{f}\right) e_{4} \\
& +\beta_{31} \varepsilon \sin (\omega t) \sin \left(e_{3}\right)+\beta_{32} \varepsilon \sin (\omega t) \sin \left(e_{3}-e_{1}\right)
\end{aligned}
$$

then

$$
\dot{V}(e)=-e_{1}^{2}-e_{2}^{2}-e_{3}^{2}-e_{4}^{2},
$$

$\dot{V}(e)<0$ is satisfied. Since $\dot{V}(e)$ is a negatively defined function, the error states are $\lim _{t \rightarrow \infty} e(t)=0$. Thus, the states of the controlled response system and drive system are synchronized globally and asymptotically [36].

The correctness of these theoretical results was evaluated using simulations with the following perturbation parameters: $\varepsilon=0.05$ and $\omega=125.6 \mathrm{rad} / \mathrm{s}$. The results are, respectively, presented in Figures 11(a)-11(d), as follows: $e_{1}=z_{1}-y_{1}$, $e_{2}=z_{2}-y_{2}, e_{3}=z_{3}-y_{3}$, and $e_{4}=z_{4}-y_{4}$. The synchronization error eventually converges to zero, resulting in stabilization of the error system. This is a clear demonstration that despite parametric perturbations, the controller is able to synchronize the states of the drive and response systems. In other words, the proposed control method is robust to parameter mismatch in the power system. The bifurcation diagram in Figure 12 was also used to illustrate the effects of parametric perturbation. Again, the proposed control method was shown to suppress chaotic behavior under the perturbed parameters (e.g., $\beta_{21}, \beta_{23}, \beta_{31}, \beta_{32}, D_{2}, D_{3}, P_{2}$, and $\left.\bar{P}_{3}\right)$ in the periodic excitation using equations (14a)-(14d).

\section{Conclusions}

Phase portraits, Poincaré maps, and frequency spectra were used to explore the rich nonlinear dynamics in power systems as well as methods used to control chaos. Bifurcation diagrams revealed several nonlinear behaviors associated with the emergence of chaotic motion at lower values of $K_{f}$. We also observed a cascade of period-doubling bifurcations prior to the onset of chaos. The most powerful approach to predicting chaotic motion involves computing the Lyapunov exponent and Lyapunov dimension. We developed a continuous feedback-control method based on synchronization for suppressing chaotic motion in power systems. The robustness of parametric perturbation on a power system with synchronization control was analyzed using bifurcation diagrams and Lyapunov stability theory. We believe that efforts to control chaos based on nonlinear dynamics could help to prevent voltage collapse in power systems.

\section{Data Availability}

All data generated or analyzed during this study are included within this article.

\section{Conflicts of Interest}

The author declares that there are no conflicts of interest regarding the publication of this paper.

\section{Acknowledgments}

This research was supported by the Ministry of Science and Technology in Taiwan, Republic of China, under project number MOST 108-2221-E-212-010-MY3.

\section{References}

[1] Md. K. M. Rabby, A. H. Chowdhury, Md. A. Azamand, and Md. A. Towfiq, "Bifurcation analysis to identify voltage collapse in Bangladesh power system," in Proceedings of the International Conference on Informatics, Electronics and Vision (ICIEV), pp. 1-5, Dhaka, Bangladesh, May 2013.

[2] J. W. Simpson-Porco and F. Bullo, "Distributed monitoring of voltage collapse sensitivity indices," IEEE Transactions on Smart Grid, vol. 7, no. 4, pp. 1979-1988, 2016.

[3] S. Yari and H. Khoshkhoo, "Assessment of line stability indices in detection of voltage stability status," in Proceedings of the IEEE International Conference on Environment and Electrical Engineering and IEEE Industrial and Commercial Power Systems Europe (EEEIC/I\&CPS Europe), pp. 1-5, Milan, Italy, June 2017.

[4] I. A. Samuel, J. Katende, C. O. A. Awosope, and A. A. Awelewa, "Prediction of voltage collapse in electrical power system networks using a new voltage stability index," International Journal of Applied Engineering Research, vol. 12, no. 2, pp. 190-199, 2017. 
[5] H. Ma, F. Min, and Y. Wang, "Nonlinear dynamic analysis and surface sliding mode controller based on low pass filter for chaotic oscillation in power system with power disturbance," Chinese Journal of Physics, vol. 56, no. 5, pp. 2488-2499, 2018.

[6] E. Ott, C. Grebogi, and J. A. Yorke, "Controlling chaos," Physical Review Letters, vol. 64, no. 11, pp. 1196-1199, 1990.

[7] H. Ren and D. Liu, "Nonlinear feedback control of chaos in permanent magnet synchronous motor," IEEE Transactions on Circuits and Systems II: Express Briefs, vol. 53, no. 1, pp. $45-50,2006$.

[8] R. Wang and Z. Jing, "Chaos control of chaotic pendulum system," Chaos, Solitons \& Fractals, vol. 21, no. 1, pp. 201-207, 2004.

[9] M. J. Liu and Z. L. Piao, "Study on chaos control for nonlinear power system," in Proceedings of the IEEE International Workshop on Intelligent Systems and Applications, pp. 1-4, Wuhan, China, May 2009.

[10] M. Kumar and P. P. Singh, "Chaos control of an extended four dimensional fundamental power system using sliding mode control," in Proceedings of the 5th IEEE Uttar Pradesh Section International Conference on Electrical, Electronics and Computer Engineering (UPCON), pp. 1-6, Gorakhpur, India, November 2018.

[11] T. L. Carroll and L. M. Pecora, "Synchronizing chaotic circuits," IEEE Transactions on Circuits and Systems, vol. 38, no. 4, pp. 453-456, 1991.

[12] J. K. John and R. E. Amritkar, "Synchronization by feedback and adaptive control," International Journal of Bifurcation and Chaos, vol. 04, no. 06, pp. 1687-1695, 1994.

[13] S. Li and Y. P. Tian, "Finite time synchronization of chaotic systems," Chaos, Solitons \& Fractals, vol. 15, no. 2, pp. 3030-3310, 2003.

[14] E.-w. Bai and K. E. Lonngren, "Synchronization and control of chaotic systems," Chaos, Solitons \& Fractals, vol. 10, no. 9, pp. 1571-1575, 1999.

[15] L. M. Pecora and T. L. Carroll, "Synchronization in chaotic systems," Physical Review Letters, vol. 64, no. 8, pp. 821-824, 1990.

[16] T.-L. Liao and S.-H. Tsai, "Adaptive synchronization of chaotic systems and its application to secure communications," Chaos, Solitons \& Fractals, vol. 11, no. 9, pp. 1387-1396, 2000.

[17] K. Pyragas, "Continuous control of chaos by self-controlling feedback," Physics Letters A, vol. 170, no. 6, pp. 421-428, 1992.

[18] T. Kapitaniak, "Continuous control and synchronization in chaotic systems," Chaos, Solitons \& Fractals, vol. 6, pp. 237244, 1995.

[19] S. C. Chang and J. F. Hu, "Codimension-two bifurcation analysis and chaos synchronization of a quarter-car vehicle model," Advances in Mechanical Engineering, vol. 10, no. 4, pp. 1-14, 2018.

[20] S.-C. Chang and Y.-F. Lue, "A study of the nonlinear response and chaos suppression in a magnetically levitated system," Australian Journal of Mechanical Engineering, vol. 18, no. 1, pp. 94-105, 2020.

[21] M. Yue and R. Schlueter, "Bifurcation subsystem and its application in power system analysis," IEEE Transactions on Power Systems, vol. 19, no. 4, pp. 1885-1893, 2004.

[22] G. Revel, A. E. Leon, D. M. Alonso, and J. L. Moiola, "Bifurcation analysis on a multimachine power system model," IEEE Transactions on Circuits and Systems I: Regular Papers, vol. 57, no. 4, pp. 937-949, 2010.
[23] H. Gholizadeh, A. Hassannia, and A. Azarfar, "Chaos detection and control in a typical power system," Chinese Physics $B$, vol. 22, no. 1, Article ID 010503, 2013.

[24] M. L. Ma and F. H. Min, "Bifurcation behavior and coexisting motions in a time-delayed power system," Chinese Physics B, vol. 24, no. 3, Article ID 030501, 2015.

[25] X. Wang, Y. Chen, G. Han, and C. Song, "Nonlinear dynamic analysis of a single-machine infinite-bus power system," Applied Mathematical Modelling, vol. 39, no. 10-11, pp. 2951-2961, 2015.

[26] P. M. Vahdati and A. Kazemi, "Bifurcations and chaos in nonlinear dynamics of power systems," in Proceedings of the 24th Iranian Conference on Electrical Engineering (ICEE), pp. 1706-1711, Shiraz, Iran, May 2016.

[27] K. Rajagopal, A. Karthikeyan, P. Duraisamy, R. Weldegiorgis, and G. Tadesse, "Bifurcation, chaos and its control in a fractional order power system model with uncertainties," Asian Journal of Control, vol. 21, no. 1, pp. 184-193, 2019.

[28] A. Wolf, J. B. Swift, H. L. Swinney, and J. A. Vastano, "Determining Lyapunov exponents from a time series," Physica D: Nonlinear Phenomena, vol. 16, no. 3, pp. 285-317, 1985.

[29] M. Rafikov and J. M. Balthazar, "On an optimal control design for Rossler system," Physics Letters A, vol. 333, no. 3-4, pp. 241-245, 2004.

[30] M. Rafikov and J. M. Balthazar, "On control and synchronization in chaotic and hyperchaotic systems via linear feedback control," Communications in Nonlinear Science and Numerical Simulation, vol. 13, no. 7, pp. 1246-1255, 2008.

[31] M. Rafikov, J. M. Balthazar, and A. M. Tusset, "An optimal linear control design for nonlinear systems," Journal of the Brazilian Society of Mechanical Sciences and Engineering, vol. 30, no. 4, pp. 279-284, 2008.

[32] N. Kopell and R. Washburn, "Chaotic motions in the twodegree-of-freedom swing equations," IEEE Transactions on Circuits and Systems, vol. 29, no. 11, pp. 738-746, 1982.

[33] S. C. Chang, Study of Structural Stability for Power System, Master Thesis, National Central University, Taoyuan City, Taiwan, 1992.

[34] IMSL Inc, User's Manual, IMSL Math/Library, Minneapolis, MN, USA, 1989.

[35] J. L. Kaplan and L. A. York, "Chaotic behavior of multidimensional difference equations," in Lecture Notes in MathematicsSpringer-Verlag, New York, NY, USA, 1979.

[36] H. K. Khalil, Nonlinear Systems, Prentice-Hall, Upper Saddle River, NJ, USA, 2002. 